\title{
Conformal Representation of Multiply Connected Domain on Many-sheeted Disc.
}

\author{
Akira MoRI.
}

(Received Oct. 1, 1949)

Bieberbach" $^{1)}$ proved first, that any schlicht domain $D$ bounded by $p$ continua can be conformally mapped on a $p$-sheeted unit disc, i. e. on a Riemann surface, which covers each point inside the unit circle exactly p-times. Afterwards Grunsky ${ }^{2}$ gave another proof of this theorem and added that this mapping is uniquely determinate under the condition mentioned later in Theorem 1.

In this paper we shall treat more generally the conformal mapping of $D$ on a $k$-sheeted unit disc, where $k$ is any integer $\geqq p$, and prove some results concerning this mapping. But since the general case $k \geqq p$ can be discussed quite the same, we will, for simplicity, confine ourselves to the case $k=p$ for a while.

Without loss of generality we can assume that $D$ lies in the finite part of the $x$-plane, and is bounded by $p$ closed analytic Jordan curves $\Gamma_{1} \ldots \ldots$, $\Gamma_{p}$. We denote by $g\left(x, x^{\prime}\right)$ the Green's function of $D$ with $x^{\prime}$ as its pole, and by $h\left(x, x^{\prime}\right)$ the conjugate function of $g\left(x, x^{\prime}\right)$. For $\lambda=1, \ldots \ldots$, $p$, let $u_{\lambda}(x)$ be the harmonic measure of the boundary curve $\Gamma_{\lambda}$ with respect to $D$, and $w_{\lambda}(x)=u_{\lambda}(x)+i v_{\lambda}(x)$ be the regular function with the real part $u_{\lambda}(x) .2 v_{\lambda}(x)$ is regular on the closure $\bar{D}$ of $D$, and, if $p>1$, infinitely many-valued.

We use the following two lemmas several times in the sequel.

Lemma 1. Let $x_{1}, \ldots \ldots, x_{p}$ le $p$ points in $D$, If these points satisfy the $p$ equations :

$$
\sum_{\mu=1}^{p} u_{\lambda}\left(x_{\mu}\right)=1 \quad(\lambda=1, \ldots \ldots, p),
$$

and only in such a case, there exists a function $y=y(x)$, which maps $D$ conformally on a p-sheeted unit disc $\Delta$ on $y$-plane, so that the images of $x_{1} .$. $\ldots x_{p}$ on $\Delta$ have one and the same projection $y=0$. In this case, the mapping function is given by 


$$
y=y(x)=\varepsilon \cdot \exp \cdot\left\{-\sum_{\mu=1}^{p}\left(\left(g\left(x, x_{\mu}\right)+i h\left(x, x_{\mu}\right)\right)\right\},|\varepsilon|=1\right.
$$

If some of the points $x_{\mu}$ coincide, e. $\mathrm{g}$. if $x_{1}=x_{2}=\ldots \ldots=x_{r+1}$, the statement of the lemma means that the image of $x_{1}=\ldots \ldots=x_{r+1}$ on $\Delta$ is a branch-point of order $r$ lying on $y=0$.

Proof. Sufficiency. It suffices to show that

$$
-\sum_{\mu=1}^{p} h\left(x, x_{\mu}\right)
$$

has the modulus of periodicity $2 \pi$ around each $\Gamma_{\lambda}$, and this follows immediately from the well-known formulae

$$
-\int_{\Gamma_{\lambda}} d h\left(x, x_{\mu}\right)=2 \pi u_{\lambda}\left(x_{\mu}\right) \quad(\lambda, \mu=1, \ldots \ldots, p)
$$

and (1), by summing up for $\mu=1, \ldots \ldots, p$. Necessity. Since

$$
\log |y(x)|+\sum_{\mu=1}^{p} g\left(x, x_{\mu}\right)
$$

is harmonic in $D$ and vanishes on the boundary of $D$, it must be identically zero, so that $y=y(x)$ is expressed in the form (2). Then (3) must have modulus of periodicity $2 \pi$ around each $\Gamma_{\lambda}$, so that, by (4), (1) holds, q. e. d.

Remark. By means of a linear transformation of $y$-plane, we see easily from Lemma 1 that, if $D$ is conformally mapped on a $p$-sheeted unit disc, and if the images of $x_{1} \ldots \ldots, x_{p}$ have one and the same projection on the plane, then necessarily (1) holds.

Lemma 2. Suppose that $D$ is conformally mapped on a p-sheeted unit disc by a function $y=y(x)$, and let $x_{1}(y), \ldots \ldots, x_{p}(y)$ denote the $p$ branches of the inverse function of $y=y(x)$. Then zee have, for any two points $y_{0}, y$ in $|y| \leqq 1$,

$$
W_{\lambda}\left(y, y_{0}\right)=\sum_{\mu=1}^{p} \int_{x_{\mu}\left(y_{0}\right)}^{x_{\mu}(y)} d z v_{\lambda}=0 \quad(\lambda=1, \ldots \ldots, p)
$$

where the paths of integration are $p$ curves in $\bar{D}$, which are described by $x_{1}(y), \ldots . ., x_{p}(y)$ when $y$ moves along a curve in $|y| \leqq 1$ from $y_{0}$ to $y$. 
Proof. By the above remark, we have

$\mathfrak{R} W_{\lambda}\left(y, y_{0}\right) \equiv \sum_{\mu=1}^{p} u_{\lambda}\left(x_{\mu}(y)\right)-\sum_{\mu=1}^{p} u_{\lambda}\left(x_{\mu}\left(y_{0}\right)\right) \equiv 1-1 \equiv 0 \quad(\lambda=1, \ldots \ldots, p)$.

Since $W_{\lambda}\left(y, y_{0}\right)$ are analytic functions of $y$ in $|y| \leqq 1$, and vanish at $y=y_{0}$, they must vanish identically, q. e. d.

We will prove

Theorem 1. (Bieberbach-Grunsky). Let $\xi_{\mu}$ be a point on $\Gamma_{\mu}$ respectivily for $\mu=1, \ldots . ., p$. Then there exists a function $y=y(x)$, which maps $D$ conformally on a p-sheeted unit disc $\Delta$ on $y$-plane, so that the images of $\tilde{s}_{1}, \ldots$ $\ldots, \xi_{p}$ on $\Delta$ have one and the same projection on $|y|=1$. This mapping function is uniquely determinate, save for a linear transformation of $y$-plane, which makes $|y|<1$ invariant.

Proof. First we will prove the existence of a system of $p$ points in $D$ satisfying (1). Since $\xi_{1}, \ldots \ldots, \xi_{p}$ obviously satisfy $(1),(1)$ is equivalent with

$$
\sum_{\mu=1}^{p}\left\{u_{\lambda}\left(x_{\mu}\right)-u_{\lambda}\left(\xi_{\mu}\right)\right\}=0 \quad(\lambda=1, \ldots \ldots, p)
$$

Instead of these equations, we consider, together with their imaginary parts,

$$
W_{\lambda}=\sum_{\mu=1}^{p} \int_{\xi \mu}^{x_{\mu}} d z w_{\lambda}=0 \quad(\lambda=1, \ldots, \ldots, p)
$$

Since we have $\sum_{1}^{p} u_{\lambda} \equiv 1$, any one of $(5)$, e. $g$. the one for $\lambda=1$, necessarily holds, if the other $p-1$ equations hold. Hence, in order to prove that (5) has solutions for a value of $x_{1}$ lying in a neighbourhood of $\xi_{1}$, we have only to show that the Jacobian of $W_{2}, \ldots . ., W_{p}$ with respect to $p-1$ variables $x_{2}, \ldots . ., x_{p}$ does not vanish at $x_{1}=\xi_{1}, x_{2}=\xi_{2}, \ldots \ldots, x_{p}=\xi_{p}$, i. e.

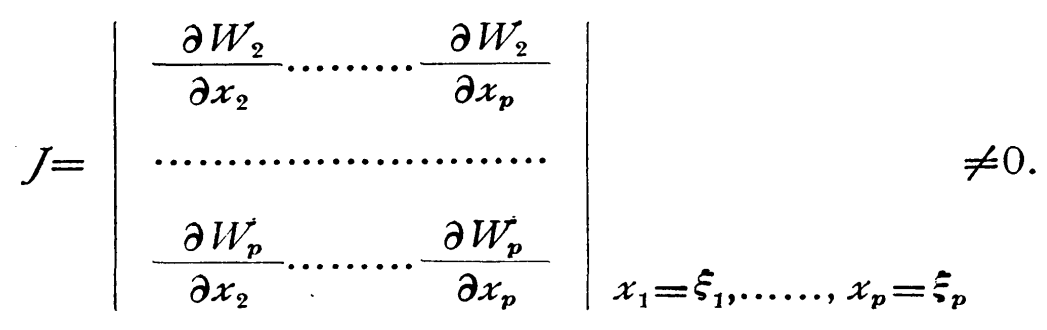


Let $\theta_{\mu}$ be the angle between the positive real axis and the positive tangent of $I_{\mu}^{\prime}$ at $\xi_{\mu}$. Then, denoting by $n, s$ the inner normal and the length of $\Gamma_{\mu}$, we have

$$
\begin{aligned}
& \left\{\frac{\partial W_{\lambda}}{\partial x_{\mu}}\right\}_{x_{1}=\xi_{1}, \ldots, x_{p}=\xi_{p}}=\left\{\frac{d}{d x} w_{\lambda}\right\}_{\xi_{\mu}}= \\
& =e^{i \theta_{\mu}}\left\{\frac{\partial}{\partial s} u_{\lambda}+i \frac{\partial}{\partial s} v_{\lambda}\right\}_{\xi_{\mu}}=e^{i \theta_{\mu}}\left\{i \frac{\partial}{\partial s} v_{\lambda}\right\}_{\xi_{\mu}}=-i e^{i \theta_{\mu}}\left\{\frac{\partial}{\partial n} u_{\lambda}\right\}_{\xi_{\mu}},
\end{aligned}
$$

so that

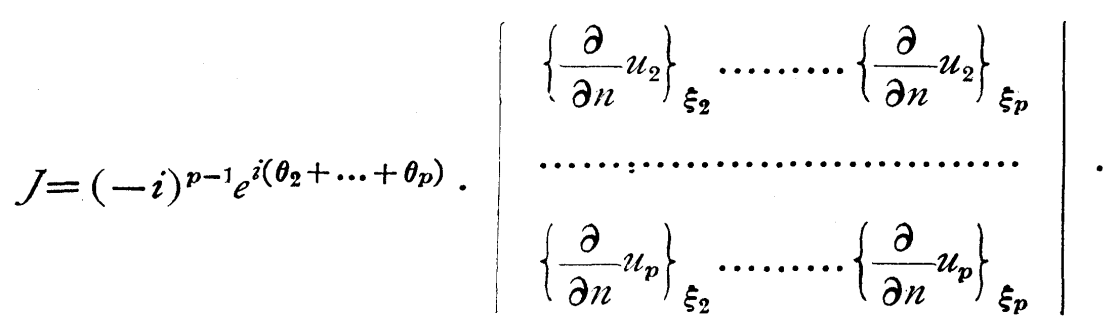

Suppose that $J=0$, then we can find $p-1$ real numbers $c_{2}, \cdots \cdots, c_{p}$, at least one of which is $>0$, such that

$$
\sum_{\lambda=2}^{p} c_{\lambda}\left\{\frac{\partial}{\partial n} u_{\lambda}\right\}_{\xi \mu}=\left\{\frac{\partial}{\partial n} \sum_{\lambda=2}^{p} c_{\lambda} u_{\lambda}\right\}_{\xi \mu}=0 \quad(\mu=2, \cdots, p) .
$$

hold. Let $c_{m}$ be the greatest of $c_{2}, \cdots \cdots, c_{p}$, then since

$$
\left\{\frac{\partial}{\partial s} \sum_{\lambda=2}^{p} c_{\lambda} u_{\lambda}\right\}_{\xi_{m}}=0
$$

we have

$$
\left\{\frac{d}{d x} \sum_{\lambda=2}^{p} c_{\lambda} w_{\lambda}\right\}_{\xi_{m}}=0
$$

So that the niveau curve $\mathfrak{R} \sum_{2}^{p} c_{\lambda} z v_{\lambda}=\sum_{2}^{p} c_{\lambda} u_{\lambda}=c_{m}$ has a multiple point at $\xi_{m}$. Then the harmonic function $\sum_{: 2}^{p} c_{\lambda} u_{\lambda}$ attains its maximum $c_{m}$ at an interior point of $D$. Hence, by the maximum principle, we have

$$
\sum_{\lambda=2}^{p} c_{\lambda} u_{\lambda}(x) \equiv c_{m}>0
$$

which contradicts the fact that the left-hand side vanishes on $\Gamma_{1}$, hence $J \neq 0$. 
Since $J \neq 0$, we can solve the simultaneous equations (5) for any $x_{1}$ lying in a certain neighbourhood $U_{1}$ of $\xi_{1}$, and obtain $p-1$ regular analytic functions of $x_{1} \in U_{1}$ :

$$
x_{2}=\varphi_{2}\left(x_{1}\right), \cdots \cdots, x_{p}=\varphi_{p}\left(x_{1}\right) \text {, }
$$

whose values lie respectively in certain neighbourhoods $U_{2}, \ldots, \ldots, U_{p}$ of $\xi_{2}, \ldots \ldots, \xi_{p}$. And the system

$$
x_{1}, \varphi_{2}\left(x_{1}\right), \ldots \ldots, \varphi_{p}\left(x_{1}\right)
$$

exhausts all the solutions of $(5)$, which lie respectively in $U_{1}, \ldots . ., U_{p}$ together with paths of integration from $\xi_{1} \cdots \cdots, \xi_{p}$. Especially for $x_{1}=\xi_{1}$, we have

$$
\varphi_{2}\left(\xi_{1}\right)=\xi_{2}, \ldots, \varphi_{p}\left(\xi_{1}\right)=\xi_{p}
$$

Further, since $\frac{d}{d x} w_{\lambda}$ does not vanish on the boundary of $D$, and the neighbourhoods $U_{1}, \ldots . ., U_{p}$ can be taken as small as we please, we can assume that

$$
u_{\lambda}(x) \leqq 0 \text { for } x \text { lying in } U_{\mu}(\mu \neq \lambda) \text { but outside } D \text {. }
$$

We fix a point $\xi_{1}^{*}$ in the common part of $U_{1}$ and $D$ arbitrarily, and put

$$
\varphi_{2}\left(\xi_{1}^{*}\right)=\xi_{2}^{*}, \ldots, \ldots, \varphi_{p}\left(\xi_{2}^{*}\right)=\xi_{p}^{*}
$$

so that we have

$$
\sum_{\mu=1}^{p} \int_{\xi \mu}^{\xi \mu^{*}} d z v_{\lambda}=0 \quad(\lambda=1, \cdots \cdots, p)
$$

with paths of integration contained respectively in $U_{1}, \cdots \cdots, U_{p}$, and consequently

$$
\sum_{\mu=1}^{p} u_{\lambda}\left(\xi_{\mu}^{*}\right)=\sum_{\mu=1}^{p} u_{\lambda}\left(\xi_{\mu}\right)=1 \quad(\lambda=1, \cdots \cdots, p) .
$$

We will prove that $\xi_{\mu}^{*}(1 \leqq \mu \leqq p)$ lies in $D$. Suppose that $\xi_{1}^{*}, \ldots . ., \xi_{h}^{*} \epsilon D$ and $\xi_{h+1}^{*}, \ldots \ldots, \xi_{p}^{*} \notin D(1 \leqq h<p)$ by suitable change. of indices. Then, since 


$$
\sum_{\lambda=1}^{h} u_{\lambda}(x)<1 \text { in } D
$$

we have, by summing up for $x=\xi_{1}^{*}, \ldots \ldots, \xi_{h}^{*}$,

$$
\sum_{\mu=1}^{h}\left(\sum_{\lambda=1}^{h} u_{\lambda}\left(\boldsymbol{\xi}_{\mu}^{*}\right)\right)<h \text {. }
$$

On the other hand, we have, by summing up (8) for $\lambda=1, \ldots \ldots, h$,

$$
\sum_{\mu=1}^{p}\left(\sum_{\lambda=1}^{h} u_{\lambda}\left(\xi_{\mu}^{*}\right)\right)=\sum_{\lambda=1}^{h}\left(\sum_{\mu=1}^{p} u_{\lambda}\left(\xi_{\mu}^{*}\right)\right)=h .
$$

Hence there exists at least one index $\mu=m$ greater than $h$, such that

$$
\sum_{\lambda=1}^{h} u_{\lambda}\left(\xi_{m}^{*}\right)>0
$$

Then, since $\xi_{m}^{*}$ lies in $U_{m}(m>h)$, it follows from $(6)$ that $\xi_{m}^{*}$ lies in $D$. This contradicts the hypothesis and proves our assertion.

Hence, if we put

$$
y=y(x)=\exp \cdot\left\{-\sum_{\mu=1}^{p}\left(g\left(x, \xi_{\mu}^{*}\right)+i h\left(x, \xi_{\mu}^{*}\right)\right)\right\}
$$

then, by Lemma $1, y=y(x)$ maps $D$ conformally on a p-sheeted unit disc $\Delta$ on $y$-plane.

Next we will prove that $\xi_{1}, \ldots . ., \xi_{p}$ are mapped on one and the same point on $|y|=1$ by $y=y(x)$. Let

$$
x_{1}(y), x_{2}(y), \ldots \ldots, x_{p}(y)
$$

be the $p$ branches of the inverse function of $y=y(x)$ determined by

$$
x_{1}(0)=\xi_{1}^{*} \in U_{1}, x_{2}(0)=\xi_{2}^{*} \in U_{2}, \cdots, x_{p}(0)=\xi_{p}^{*} \in U_{p} .
$$

Then, if $y$ lies in a sufficiently small neighbourhood $V$ of $y=0$, the values of (9) fall respectively in $U_{1}, U_{2}, \cdots \cdots, U_{p}$, so that, by Lemma 2 , we have for $y$ lying in $V$ together with a path from $y=0$

$$
\sum_{\mu=1}^{p} \int_{\xi \mu^{*}}^{x_{\mu}(y)} d w_{\lambda}=0 \quad(\lambda=1, \cdots \cdots, p)
$$


A. Mori.

with paths of integration contained respectively in $U_{1}, \cdots \cdots, U_{p}$. Hence, by $(7)$, we obtain

$$
\sum_{\mu=1}^{p} \int_{\xi_{i \mu}}^{x_{\mu}(y)} d w_{\lambda}=0 \quad(\lambda=1, \cdots \cdots, p)
$$

i. e. (9) provides a system of solutions of the equations (5), which lie respectively in $U_{1}, \cdots \cdots, U_{p}$ together with paths of integration, if $y \in V$. Then, by the uniqueness of solutions of (5) in $U_{1}, \ldots, \ldots, U_{p}$, if we put

$$
x_{1}(y)=x_{1} \quad \text { i. e. } y=y\left(x_{1}\right) \text {, }
$$

we have

$$
x_{2}(y)=\varphi_{2}\left(x_{1}\right), \cdots \cdots, x_{p}(y)=\varphi_{p}\left(x_{1}\right)
$$

for $x_{1}$ lying in a sufficiently small neighbourhood of $\xi_{1}^{*}$, such that $y=y\left(x_{1}\right)$ falls in $V$. But since the both sides of (11) are analytic functions of $x_{1} \in U_{1}$ in virtue of (10), (11) holds throughout $U_{1}$. Hence, especially for $x_{1}=\xi_{1}$, we have, putting $\eta=y\left(\xi_{1}\right)$,

$$
x_{2}(\eta)=\varphi_{2}\left(\xi_{1}\right)=\xi_{2}, \cdots \cdots, x_{p}(\eta)=\varphi_{p}\left(\xi_{1}\right)=\xi_{p},
$$

so that

$$
r_{1}=y\left(\xi_{1}\right)=y\left(\xi_{2}\right)=\cdots \cdots=y\left(\xi_{p}\right)
$$

Finally we will prove the uniqueness of the mapping. function. Let $y^{\prime}=y^{\prime}(x)$ be another mapping function satisfying the condition of the theorem. By means of a linear transformation of $y^{\prime}$-plane, we can assume that $y^{\prime}\left(\boldsymbol{\xi}_{1}^{*}\right)=0$, and then, by the expression for the mapping function given in Lemma 1, it suffices to prove that the $p$ zero points of $y^{\prime}=y^{\prime}(x)$ coincide with those of $y=y(x)$ constructed above, i. e.

$$
y^{\prime}\left(\xi_{1}^{*}\right)=y^{\prime}\left(\xi_{2}^{*}\right)=\ldots \ldots=y^{\prime}\left(\xi_{p}^{*}\right)=0 .
$$

Let $\eta^{\prime}$ be the value of $y^{\prime}(x)$ at $\xi_{1}, \cdots \cdots, \xi_{p}$, and let

$$
x_{1}^{\prime}\left(y^{\prime}\right), x_{2}^{\prime}\left(y^{\prime}\right), \ldots \ldots, x_{p}^{\prime}\left(y^{\prime}\right)
$$

be the $p$ branches of the inverse function of $y^{\prime}=y^{\prime}(x)$ determined by 


$$
x_{1}^{\prime}\left(\eta^{\prime}\right)=\xi_{1}, x_{2}^{\prime}\left(\eta^{\prime}\right)=\tilde{\xi}_{2}, \ldots \ldots, x_{p}^{\prime}\left(\eta^{\prime}\right)={ }_{p} .
$$

Then, as before by Lemma 2, (13) provides a system of solutions of $(5)$ lying respectively in $U_{1}, \ldots \ldots, U_{p}$ together with paths of integration, if $y^{\prime}$ lies in a sufficiently small neighbourhood of $y^{\prime}=\eta^{\prime}$. Then, by the uniqueness of solutions of $(5)$ in $U_{1}, \ldots \ldots, U_{p}$, if we put $x_{1}^{\prime}\left(y^{\prime}\right)=x_{1}$ i. e. $y^{\prime}=y^{\prime}\left(x_{1}\right)$, we have

$$
x_{2}^{\prime}\left(y^{\prime}\right)=\varphi_{2}\left(x_{1}\right), \ldots \ldots, x_{p}^{\prime}\left(y^{\prime}\right)=\varphi_{p}^{\prime \prime}\left(x_{1}\right)
$$

which hold throughout $U_{1}$ as before. Especially for $x_{1}=\xi_{1}^{*}$, we obtain, by $y^{\prime}=y^{\prime}\left(\xi_{1}^{*}\right)=0$,

$$
x_{2}^{\prime}(0)=\varphi_{2}\left(\xi_{1}^{*}\right)=\xi_{2}^{*}, \ldots, \ldots, x_{p}^{\prime}(0)=\varphi_{p}\left(\xi_{1}^{*}\right)=\xi_{p}^{*},
$$

from which (12) follows. Thus the theorem is completely proved.

Next, we will prove

Theorem 2. Let $x_{1}, \ldots \ldots, x_{p}$ be $p$ points in $\bar{D}$. Then, in order that. the images of these points by the conformal mapping of Theorem 1 have one and the same projection on $y$-plane, it is necessary and sufficient that the $p$ equations:

$$
\sum_{\mu=1}^{p} \int_{\xi_{\mu}}^{x_{\mu}} d w_{\lambda}=0 \quad(\lambda=1, \cdots \cdots, p)
$$

simultaneously hold, with certain paths of integration in $\bar{D}$ independent of $\lambda$.

Proof. Necessity. In the first place, we connect the common projection of the images of $x_{1}, \cdots \cdots, x_{p}$ with that of $\xi_{1}, \cdots \cdots, \xi_{p}$ by a curve in $|y| \leqq 1$. Then correspoding to this curve we obtain, by Lemma $2, p$ paths of integration in $\bar{D}$, such that

$$
\sum_{\mu=1}^{p} \int_{\xi_{\mu}}^{x_{\mu^{\prime}}} d w_{\lambda}=0 \quad(\lambda=1, \cdots \cdots, p)
$$

hold, where $\left(x_{1}^{\prime}, \cdots \cdots, x_{p}^{\prime}\right)$ is a permutation of $\left(x_{1}, \cdots \cdots, x_{p}\right)$. Next, we can apply any transposition to $\left(x_{1}^{\prime} \ldots \ldots, x_{p}^{\prime}\right)$, while we add to (15) two integrals taken in positive and negative directions over a curve in $\bar{D}$, which connects any two of $x_{1}^{\prime} \cdots \cdots x_{p}^{\prime}$. Since any permutation can be expressed as product of several number of transpositions, we obtain the result.

Sufficiency. Since we have from (14) 


$$
\sum_{\mu=1}^{p} u_{\lambda}\left(x_{\mu}\right)=\sum_{\mu=1}^{p} u_{\lambda}\left(\xi_{\mu}\right)=1 \quad(\lambda=1, \cdots \cdots, p)
$$

and since $x_{1}, \ldots \ldots, x_{p}$ lie in $\bar{D}$, it is easily seen that only two cases are possible, viz. (i) $x_{1}, \ldots \ldots, x_{p}$ lie on the boundary of $D$ respectively one on each boundary curve, or (ii) any one of $x_{1}, \ldots . ., x_{p}$ lies in $D$. We will first prove the theorem for the case (i).

Let $C_{1}, \ldots \ldots, C_{p}$ be the $p$ paths of integration in $\bar{D}$, which connect respectively $x_{\mu}$ with $\xi_{\mu}$, and $x_{\mu}^{\prime}$ be the one of $x_{1}, \ldots \ldots x_{p}$, which lies on $\Gamma_{\mu}$. If we denote by $\gamma_{\mu}$ the positive arc of $I_{\mu}$ from $\xi_{\mu}$ to $x_{\mu}^{\prime}$, then the curves $C_{1}, \ldots \ldots C_{p}$, together with $\gamma_{1}, \ldots \ldots, \gamma_{p}$ taken in negative direction, make up a several number of closed curves $C$ in $D$. Hence, we can find $p$ integers $r_{1}, \ldots \ldots, r_{p}$, such that $C$ is homologous to $r_{1} \Gamma_{1}+\ldots \ldots+r_{p} \Gamma_{p}$, i. e.

$$
C=C_{1}+\ldots \ldots+C_{p}-r_{1}-\ldots \ldots-r_{p} \sim r_{1} \Gamma_{2}+\ldots \ldots+r_{p} \Gamma_{p}^{\prime},
$$

so that the paths of integration $C_{1}+\ldots \ldots+C_{p}$ can be replaced by $\left(\gamma_{1}+r_{1} \Gamma_{1}\right)+$ $\ldots \ldots+\left(\gamma_{p}+r_{p} \Gamma_{p}^{\prime}\right)$, and we obtain

$$
\sum_{\mu=1}^{p} \int_{\gamma \mu+r_{\mu} \Gamma_{\mu}} d w_{\lambda}=0 \quad(\lambda=1, \ldots \ldots, p) .
$$

Since $\Gamma_{1}+\ldots \ldots+\Gamma_{p}^{\prime} \sim 0$, we can assume that $r_{\mu} \geqq 0(1 \leqq \mu \leqq p)$.

By the conformal mapping of Theorem 1 , each $\gamma_{\mu}+r_{\mu} \Gamma_{\mu}$ corresponds to a positive arc $\alpha_{\mu}$ of $|y|=1$ starting from $\eta=y\left(\xi_{1}\right)=\cdots \cdots=y\left(\xi_{p}\right)$. Let $\alpha_{m}$ be the shortest of $\alpha_{1}, \cdots \cdots, \alpha_{p}$, and $\gamma_{\mu}^{\prime}$ be the part of $\gamma_{\mu}+r_{\mu} \Gamma_{\mu}$, which corresponds to $\alpha_{m}$ on $|y|=1$, so that we have, by Lemma 2,

$$
\sum_{\mu=1}^{p} \int_{\gamma \mu^{\prime}} d w_{\lambda}=0 \quad(\lambda=1, \ldots \cdots, p) \text {. }
$$

And let $\gamma_{\mu}^{\prime \prime}$ be the remaining part of $\gamma_{\mu}+r_{\mu} \Gamma_{\mu}$, so that

$$
\gamma_{\mu}^{\prime}+\gamma_{\mu}^{\prime \prime}=\gamma_{\mu}+r_{\mu} \Gamma_{\mu}
$$

and especially for $\mu=m$

$$
\gamma_{m}^{\prime}=\gamma_{m}+r_{m} \Gamma_{m}, \quad \gamma_{m}^{\prime \prime}=0 .
$$

Then, from (17), (18) and (19), we have

$$
\sum_{\mu \neq m} \int_{r_{\mu^{\prime \prime}}} d z w_{\lambda}=0 \quad(\lambda=1, \cdots \cdots, p) \text {. }
$$

If we put especially $\lambda=m$ and take the imaginary part, we obtain 


$$
\sum_{\mu \neq m} \int_{\gamma_{\mu}^{\prime \prime}} \frac{\partial u_{m}}{\partial n} d s=0
$$

Since $\frac{\partial u_{m}}{\partial n}>0$ on $\Gamma_{1}, \cdots \cdots, \Gamma_{m-1}, \Gamma_{m+1}, \cdots \cdots, \Gamma_{p}$, and since $\gamma_{1}^{\prime \prime}, \cdots \cdots, \gamma_{m-1}^{\prime \prime}$, $\gamma^{\prime \prime}{ }_{m+1}, \cdots \cdots, \gamma_{p}^{\prime \prime}$ are arcs of positive direction, it follows from (20) that $\gamma_{\mu}^{\prime \prime}=0$ for $\mu=1, \cdots \cdots, p$. Hence the arcs $u_{1}, \cdots \cdots, \alpha_{p}$ coincide, so that their ending points $i$. e. the projections of the images of $x_{1}, \ldots \ldots, x_{p}$ coincide with each others. Thus the theorem is proved for the case (i).

Next, if (ii) is the case, we can map $D$, by (16) and Lemma 1, conformally on a $p$-sheeted unit disc $\Delta^{\prime}$ by a function $y^{\prime}=y^{\prime}(x)$, so that the images of $x_{1}, \cdots \cdots, x_{p}$ on $\Delta^{\prime}$ have one and the same projection $y^{\prime}=0$. Then, while connecting $y^{\prime}=0$ with a point on $\left|y^{\prime}\right|=1$ by a curve in $\left|y^{\prime}\right| \leqq 1$, we obtain, by Lemma $2, p$ points $x_{1}^{\prime}, \ldots \ldots, x_{p}^{\prime}$ on the boundary of $D$ and $p$ paths of integration, such that

$$
\sum_{\mu=1}^{p} \int_{x_{\mu}}^{x \mu^{\prime}} d w_{\lambda}=0 \quad(\lambda=1, \cdots \cdots, p)
$$

hold, so that, by (14), we have

$$
\sum_{\mu=1}^{p} \int_{\xi \mu}^{x_{\mu^{\prime}}} d z v_{\lambda}=0 \quad(\lambda=1, \cdots \cdots, p)
$$

with certain paths of integration. Then, by the case (i) already proved, the images of $x_{1}^{\prime}, \ldots \ldots, x_{p}^{\prime}$ on $\Delta$ have one and the same projection on $|y|=1$. Hence, by the last pait of Theorem $1, y=y(x)$ is a linear function of $y^{\prime}=y^{\prime}(x)$, so that the images of $x_{1}, \cdots \cdots, x_{p}$ on $\Delta$ have also one and the same projection on $y$-plane, q. e. d.

Remark. From Theorem 1 and Theorem 2, it follows that, for any point $x_{1}$ in $D$, the simultaneous equations (14) have unique solutions $x_{2}, \cdots$ $\cdots, x_{p}$, if we neglect the paths of integration-which are independent of $\lambda$ - and the order of arrangement. $x_{2}, \ldots ., x_{p}$ are the $p-1$ points in $\bar{D}$, whose images on $\Delta$ have the same projection as that of $x_{1}$. Hence, in order that the image of $x_{1}$ on $\Delta$ be a branch-point of order $r$, it is necessary and sufficient that $r$ points, among the solutions $x_{2} \ldots \ldots, x_{p}$ of (14) for $x_{1}$, coincide with $x_{1}$.

The following theorem is an immediate consequence of Lemma 1.

Theorem 3. Let $D_{\lambda}$ be the part of $D$, such that 


$$
u_{\lambda}(x)>\frac{1}{2} \imath D_{\lambda}
$$

Then, when we map $D$ conformally on a p-sheeted unite disc $\Delta$, the image of $D_{\lambda}$ is alivays schlicht. The number $\frac{1}{2}$ can not ob replaced by any smaller.

Proof. Let $x_{1}, x_{2}$ be two points in $D_{\lambda}$. Then, since

$$
u_{\lambda}\left(x_{1}\right)+u_{\lambda}\left(x_{2}\right)>1 \text {, }
$$

the images of $x_{1}$ and $x_{2}$ on $\Delta$ can not have one and the same projection on the plane, by the remark to Lemma 1 . Next, let $D$ be the $p$-ply connected domain bounded by $\Gamma_{1}:|x|=q<1, \Gamma_{2}:|x|=1$ and $p-2$ closed curves $\Gamma_{3}, \ldots \ldots, \Gamma_{p}$ in $q<|x|<1$. For any $\varepsilon>0$, let $D_{\lambda}^{\prime}$ be the part of $D$, such that $u_{\lambda}(x)>\frac{1}{2}-\varepsilon$ in $D_{\lambda}^{\prime}$. Then, if $\Gamma_{3}, \cdots \cdots, \Gamma_{p}$ are sufficiently small and have no points in common with the circle $|x|=\sqrt{q}$, each of $D_{1}^{\prime}$ and $D_{2}^{\prime}$ contains the circle $|x|=\sqrt{q}$ wholly in it, so that the images of $D_{1}^{\prime}$ and $D_{2}^{\prime}$ on $\Delta$ can not be both schlicht, which proves the last part of the theorem.

Finally, we consider the general case $k \geqq p$. Let the boundary of $D$ be divided into $k$ arcs $\Gamma_{1}^{\prime}, \cdots \cdots, \Gamma_{k}^{\prime}$. Then, if we take these arcs, excepting both extremities, intead of $\Gamma_{1}, \ldots \ldots, \Gamma_{p}$, it is easily seen that all the above arguments subsist also in this case, though a slight modification is necessary for the proof of Theorem 2 on account of the singularities at the extremities of $\Gamma_{\lambda}^{\prime}$. E. g. to Theorem 1 corresponds

Theorem 1'. Let $\xi_{\mu}$ be an internal point of $\Gamma_{\mu}^{\prime}$ respectively for $\mu=1$, ......, $k$. Then there exists a function $y=y(x)$, wuhich maps $D$ conformally on a k-sheeted unii disc on $y$-plane, so that each $I_{\mu}^{\prime \prime}$ corresponds to one round of the unit circle, and the images of $\xi_{1}, \cdots \cdots, \xi_{k}$ have one and the same projection on $|y|=1$. Under these conditions, the mapping function is uniquely determinate, save for a linear transformation of $y$-plane, which makes $|y|<1$ invariant.

Remark. As regards the number of. branch-points of the $k$-sheeted image disc, it follows easily from the well-known Hurwitz's formula that the sum of orders of branch-points is equal to $k+p-2$.

Mathematical Institute,

Tokyo University. 


\section{References.}

1) L. Bieberbach : Über einen Riemannschen Satz aus der Lehre von der konformen Abbildung. Sitzungsber. Berliner Math. Ges. 24 (1925).

2) H. Grunsky: Über die konforme Abbildung mehrfach zusammenhängender Bereiche auf mehrblättrige Kreise. Sitzungsber. preuss. Akad. Wiss. (1937). 